\title{
Detection of Salmonella from animal sources in South Africa between 2007 and 2014
}

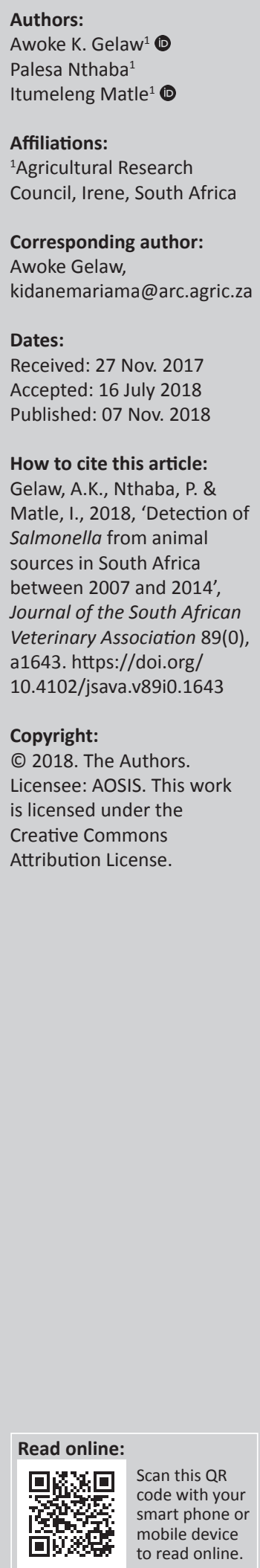

Retrospective laboratory-based surveillance was conducted on Salmonella serotypes isolated from various animal species from 2007 to 2014 at the Agricultural Research Council, Onderstepoort Veterinary Research Institute, South Africa. During the surveillance period, 1229 salmonellae isolations were recorded. Around 108 different serotypes were recovered from nine different food and non-food animal host species. The three most common serotypes were Salmonella enterica subspecies enterica serotype Heidelberg $(n=200)$, Salmonella enterica subspecies enterica serotype Enteritidis $(n=170)$ and Salmonella enterica subspecies enterica serotype Typhimurium $(n=146)$. These were followed by Salmonella enterica subspecies enterica serotype Anatum $(n=62)$ and Salmonella enterica subspecies enterica serotype Infantis $(n=57)$. Salmonella enterica subspecies enterica serotype Schwarzengrund and Salmonella enterica subspecies enterica serotype Muenchen were recovered in 50 and 48 cases, respectively. Of the total number of isolations recorded during the period under review, $871(70.8 \%)$ occurred in poultry and other birds, $162(13.2 \%)$ in horses, $116(9.4 \%)$ in cattle, $26(2.1 \%)$ in sheep and goats, $22(1.8 \%)$ in rhinoceroses, $16(1.3 \%)$ in pigs, $8(0.6 \%)$ in crocodiles, $6(0.5 \%)$ in cats and $6(0.5 \%)$ in leopards. Food animals accounted for $83.5 \%$ of the total isolations, with cattle and poultry representing approximately $72.7 \%$. Forty-two $(3.4 \%)$ isolates were found from non-food animals that include rhinoceroses $(n=22)$, crocodiles $(n=8)$, leopards $(n=6)$ and cats $(n=6)$. Salmonella Heidelberg was the most frequently isolated serotype, whereas $S$. Typhimurium had the widest zoological distribution. Clinical laboratory isolation of different Salmonella serotypes from various hosts may aid in recognising the threat to livestock, public and environmental health. Moreover, it may also highlight the potential zoonotic and food safety risk implications of the detected Salmonella serotypes.

\section{Introduction}

Salmonella infection (salmonellosis) is a common bacterial disease that mainly affects the intestinal tract of vertebrate and invertebrate hosts. Salmonella bacteria typically live in animal and human intestines and are shed in faeces. Salmonellosis remains a persistent threat to human and animal health and welfare in spite of the advances made in its detection, typing and control (Peek et al. 2004; Refsum et al. 2002). Salmonella and salmonellosis cause losses in the livestock and poultry industry because of death, abortion, decreased milk, meat and egg production, cost of testing and control programmes (Uzzau et al. 2000; Veling et al. 2002).

The genus Salmonella contains the species Salmonella enterica, Salmonella bongori and Salmonella subterranean, which was proposed in 2005 (Coburn, Grassl \& Finlay 2007). However, according to the World Health Organization, the new species, Salmonella subterranean, does not belong to the genus Salmonella (Grimont \& Weill 2007). Salmonella enterica subspecies are further classified into more than 2500 serovars or serotypes and include pathogens of great medical and veterinary importance (EFSA 2008; Mead et al. 1999; Morgan et al. 2004). These serovars differ greatly in their host range and degree of host adaptation. Salmonella serotypes are named in accordance with the Kaufmann-White typing system, defined by different combinations of somatic $\mathrm{O}$, surface $\mathrm{Vi}$ and flagellar $\mathrm{H}$ antigens, which determine the serologically defined names appended to the Salmonella species as serovars or serotypes (Popoff 2001). In 2005, Salmonella enterica finally gained official approval as the type species of the genus Salmonella (Coburn et al. 2007).

The incidence of Salmonella infections in animals is closely associated with the age of the animals, husbandry and management - related factors in intensive farming that are conducive to the spread of infection resulting in an increase of clinical disease outbreaks (Baumler et al. 1998; Kidanemariam, Engelbrecht \& Picard 2010). Clinically sick animals pose the greatest risk to 
humans because they are more likely to shed Salmonella in higher concentrations than apparently healthy animals.

Salmonellosis is one of the most common food-borne diseases. High-protein foods such as meat, poultry, fish and eggs are most commonly associated with Salmonella. However, any food that becomes contaminated and held at temperatures that promote bacterial growth can cause salmonellosis (Altekruse et al. 2006; Kidanemariam et al. 2010; Kimura et al. 2004).

Periodic surveillance reports, such as this one, could provide useful information on the changing patterns of salmonellosis in animals and foods of animal origin. It also assists in the study of the epidemiology, risk factors associated with the disease, and control of the disease. The purpose of this report is to present the retrospective records of the animal isolations and the zoological distribution of the Salmonella serotypes isolated and serotyped at the Agricultural Research Council Onderstepoort Veterinary Research (ARC-OVR) from 2007 to 2014 .

\section{Materials and methods}

Specimens submitted to the ARC-OVR bacteriology laboratory comprised bacterial cultures, organs, tissues, swabs, faeces, fluid and tissues obtained after abortion and egg samples. Unprocessed and raw specimens were inoculated into buffered peptone water $(\mathrm{pH}$ 7.2) as pre-enrichment and incubated at $37^{\circ} \mathrm{C}$ for $18-24 \mathrm{~h}$. One millilitre of the preenrichment broth was transferred into $9 \mathrm{ml}$ of Rappaport Vassiliadis $\left(\mathrm{Oxoid}^{\circledR}\right)$ enrichment broth and incubated at $42{ }^{\circ} \mathrm{C}$ for 18-24 h. Subcultures from enrichment media were grown on to selective solid media such as xylose-lysine deoxycholate (XLD) agar $\left(\right.$ Difco $\left.^{\circledR}\right)$ and incubated at $37{ }^{\circ} \mathrm{C}$ for $18-24 \mathrm{~h}$. Black colonies with a pink periphery were considered presumptively positive for Salmonella and were further confirmed with biochemical tests. Only those Gram-negative isolates that were indole-negative, motile, Simmon's citratenegative, urease negative, produced hydrogen sulphide in a triple sugar iron (TSI) slant, were lysine decarboxylase positive, fermented dulcitol but did not ferment lactose and were malonate negative were considered to be Salmonella enterica. Additional carbohydrate fermentation tests, such as gas production in Durham tubes and fermentation of sorbitol, arabinose, rhamnose, maltose and trehalose, were included to make provision for those Salmonella organisms that do not necessarily fit the above-mentioned criteria. Consideration was also given to S. enterica serovar Gallinarum and S. enterica serovar Pullorum that are non-motile when isolation and identification were done. There are variable reactions to Simmon's citrate utilisation and hydrogen production, and special attention was given to these features not to miss salmonellae.

Confirmed Salmonella isolates were further serotyped according to the Kauffmann-White classification scheme using a battery of somatic $\mathrm{O}$ and flagellar $\mathrm{H}$ polyvalent and monovalent antisera.
Data were captured in a dedicated Microsoft Excel ${ }^{\mathrm{TM}}$ data sheet for subsequent analysis. Descriptive statistics were employed to obtain values of proportions and percentiles.

\section{Results}

A total of 1229 Salmonella isolates were serotyped successfully during the surveillance period 2007-2014. The number of isolates and distribution of the various serotypes in different host species are shown in Table 1. The isolates were classified into 108 serotypes under the 17 groups of the KauffmannWhite classification scheme.

Of the total of 1229 isolates with 108 serotypes recorded during the period under review, $871(70.8 \%)$ occurred in poultry and other birds, $162(13.2 \%)$ in horses, $116(9.4 \%)$ in cattle, 26 (2.1\%) in sheep and goats, $22(1.8 \%)$ in rhinoceroses, $16(1.3 \%)$ in pigs, $8(0.6 \%)$ in crocodiles, $6(0.5 \%)$ in cats and $6(0.5 \%)$ in leopards. Despite the large number of serotypes involved, the majority of isolates were limited to only a few serotypes. For example, from the total of 108 serotypes detected, only 8 serotypes contributed $63.0 \%$ (775 isolates), while 100 serotypes collectively accounted for $37.0 \%$ (454 isolates). The eight most common serotypes were S. Anatum (5.0\%), S. Dublin (3.4\%), S. Enteritidis (13.8\%), S. Heidelberg $(16.2 \%), S$. Infantis $(4.6 \%), S$. Muenchen $(3.9 \%)$, S. Schwarzengrund $(4.0 \%)$ and $S$. Typhimurium $(11.8 \%) \quad$ (Table 1). The most frequently isolated serotype was S. Heidelberg, accounting for $16.2 \%$ of all isolates. Twenty-seven serotypes were isolated only once during the survey period. S. Typhimurium had the widest zoological distribution, followed by $S$. Enteritidis and S. Heidelberg.

In domestic fowl and other birds, the most common serotypes detected were S. Enteritidis (18.2\%), S. Heidelberg (15.2\%), S. Typhimurium (7.5\%) and S. Schwarzengrund (5.4\%); these four serotypes in total contributed $46.5 \%$ of the isolates, while the remaining 84 serotypes accounted for $53.5 \%$ of the total isolates (Tables 1 and 2).

In cattle, the most common serotypes detected were $S$. Dublin (33.6\%), S. Typhimurium (14.6\%), S. Anatum (11.2\%) and S. Infantis (6.9\%) and accounted for $66.4 \%$ of the total isolates, while the remaining 25 serotypes accounted for $33.6 \%$ of the total isolates (Table 3).

In horses, 162 isolations involving 18 serotypes were recorded in the period under review. The three major serotypes were S. Heidelberg (32.1\%), S. Anatum (22.2\%) and $S$. Typhimurium (21.6\%). The remaining 15 serotypes collectively accounted for $24.8 \%$ of the total isolations (Tables 1 and 4).

Eight serotypes were identified from 26 isolations that occurred in sheep and goats. Salmonella Typhimurium $(34.6 \%)$, S. Enteritidis $(15.4 \%)$, S. Infantis $(11.5 \%)$ and S. Isangi $(11.5 \%)$ were the major serotypes and collectively accounted for $73.1 \%$ of the isolations (Table 5). 
TABLE 1: Number of isolates and zoological distribution of the various serotypes 2007-2014.

\begin{tabular}{|c|c|c|c|c|c|c|c|c|c|c|}
\hline Serotype & Cattle & Poultry & Leopard & Rhino & Crocodile & Feline & Equine & Sheep and goat & Pig & Total \\
\hline$S$ Aarhus & 1 & 1 & 0 & 0 & 0 & 0 & 0 & 0 & 0 & 2 \\
\hline S Abaetetuba & 0 & 7 & 0 & 0 & 0 & 0 & 0 & 0 & 0 & 7 \\
\hline S Aberdeen & 3 & 9 & 0 & 0 & 0 & 0 & 0 & 0 & 0 & 12 \\
\hline S Achersleben & 0 & 0 & 0 & 0 & 0 & 0 & 2 & 0 & 0 & 2 \\
\hline S Adeoyo & 0 & 0 & 0 & 0 & 0 & 0 & 9 & 0 & 0 & 9 \\
\hline S Agona & 0 & 12 & 0 & 0 & 0 & 0 & 0 & 2 & 0 & 14 \\
\hline S Alachua & 0 & 3 & 0 & 0 & 0 & 0 & 0 & 0 & 0 & 3 \\
\hline S Anatum & 13 & 13 & 0 & 0 & 0 & 0 & 36 & 0 & 0 & 62 \\
\hline$S$ Berta & 0 & 7 & 0 & 0 & 0 & 0 & 0 & 0 & 0 & 7 \\
\hline$S$ Bovismorbificans & 1 & 0 & 0 & 0 & 0 & 0 & 0 & 0 & 0 & 1 \\
\hline S Blockley & 0 & 2 & 0 & 0 & 0 & 0 & 0 & 0 & 0 & 2 \\
\hline$S$ Brandenburg & 1 & 3 & 0 & 0 & 0 & 0 & 0 & 0 & 0 & 4 \\
\hline$S$ Braenderup & 0 & 6 & 0 & 0 & 0 & 0 & 0 & 0 & 0 & 6 \\
\hline$S$ Brancaster & 0 & 16 & 0 & 0 & 0 & 0 & 0 & 0 & 0 & 16 \\
\hline$S$ Bredeney & 1 & 3 & 0 & 0 & 0 & 0 & 0 & 0 & 0 & 4 \\
\hline S Brezany & 0 & 1 & 0 & 0 & 0 & 0 & 0 & 0 & 0 & 1 \\
\hline S Canada & 0 & 2 & 0 & 0 & 0 & 0 & 0 & 0 & 0 & 2 \\
\hline S Cardoner & 6 & 0 & 0 & 0 & 0 & 0 & 0 & 0 & 0 & 6 \\
\hline$S$ Cerro & 0 & 1 & 0 & 0 & 0 & 0 & 0 & 0 & 0 & 1 \\
\hline S Chailey & 0 & 2 & 0 & 0 & 0 & 0 & 0 & 0 & 0 & 2 \\
\hline$S$ Chester & 2 & 0 & 0 & 0 & 0 & 0 & 0 & 0 & 0 & 2 \\
\hline S Chicago & 2 & 0 & 0 & 0 & 0 & 0 & 0 & 0 & 0 & 2 \\
\hline S Cremieu & 1 & 0 & 0 & 0 & 0 & 0 & 0 & 0 & 0 & 1 \\
\hline S Claibornei & 0 & 2 & 0 & 0 & 0 & 0 & 0 & 0 & 0 & 2 \\
\hline$S$ Colorado & 0 & 0 & 0 & 0 & 0 & 0 & 4 & 0 & 0 & 4 \\
\hline$S$ Corvallis & 0 & 4 & 0 & 0 & 0 & 0 & 0 & 0 & 0 & 4 \\
\hline S Cubana & 1 & 0 & 0 & 0 & 0 & 0 & 0 & 0 & 0 & 1 \\
\hline$S$ Derby & 0 & 3 & 0 & 0 & 0 & 0 & 0 & 0 & 2 & 5 \\
\hline$S$ Dublin & 39 & 0 & 0 & 1 & 0 & 0 & 0 & 2 & 0 & 42 \\
\hline S Duesseldorf & 1 & 2 & 0 & 0 & 0 & 0 & 0 & 0 & 0 & 3 \\
\hline$S$ Duisburg & 0 & 2 & 0 & 0 & 0 & 0 & 0 & 0 & 0 & 2 \\
\hline$S$ Eastbourne & 0 & 1 & 0 & 0 & 0 & 0 & 0 & 0 & 0 & 1 \\
\hline$S$ Edinburg & 0 & 2 & 0 & 0 & 0 & 0 & 0 & 0 & 0 & 2 \\
\hline$S$ Eko & 0 & 2 & 0 & 0 & 0 & 0 & 0 & 0 & 0 & 2 \\
\hline$S$ Enteritidis & 1 & 159 & 0 & 3 & 0 & 0 & 0 & 4 & 3 & 170 \\
\hline S Farmsen & 0 & 6 & 0 & 0 & 0 & 0 & 0 & 0 & 0 & 6 \\
\hline$S$ Fulda & 0 & 4 & 0 & 0 & 8 & 0 & 0 & 0 & 0 & 12 \\
\hline$S$ Give & 0 & 0 & 0 & 0 & 0 & 0 & 0 & 0 & 1 & 1 \\
\hline S Glasgow & 0 & 0 & 0 & 0 & 0 & 0 & 2 & 0 & 0 & 2 \\
\hline S Glostrup & 0 & 1 & 0 & 0 & 0 & 0 & 0 & 0 & 0 & 1 \\
\hline$S$ Gombe & 0 & 1 & 0 & 0 & 0 & 0 & 0 & 0 & 0 & 1 \\
\hline$S$ Goldcoast & 2 & 0 & 0 & 0 & 0 & 0 & 0 & 0 & 0 & 2 \\
\hline S Gustavia & 0 & 0 & 0 & 0 & 0 & 0 & 1 & 0 & 0 & 1 \\
\hline S Hadar & 0 & 12 & 0 & 0 & 0 & 0 & 0 & 0 & 0 & 12 \\
\hline S Havana & 0 & 5 & 0 & 0 & 0 & 0 & 0 & 0 & 0 & 5 \\
\hline S Hayindogo & 0 & 4 & 0 & 0 & 0 & 0 & 0 & 0 & 0 & 4 \\
\hline S Heidelberg & 1 & 133 & 0 & 12 & 0 & 2 & 52 & 0 & 0 & 200 \\
\hline S Idikan & 0 & 3 & 0 & 0 & 0 & 0 & 0 & 0 & 0 & 3 \\
\hline S Indiana & 0 & 7 & 0 & 0 & 0 & 0 & 0 & 0 & 0 & 7 \\
\hline$S$ Infantis & 8 & 41 & 0 & 0 & 0 & 0 & 5 & 3 & 0 & 57 \\
\hline$S$ Isangi & 0 & 3 & 0 & 0 & 0 & 0 & 0 & 3 & 0 & 6 \\
\hline$S$ Israel & 0 & 1 & 0 & 0 & 0 & 0 & 0 & 0 & 0 & 1 \\
\hline S Istanbul & 0 & 1 & 0 & 0 & 0 & 0 & 0 & 0 & 0 & 1 \\
\hline$S$ Jerusalem & 0 & 2 & 0 & 0 & 0 & 0 & 0 & 0 & 0 & 2 \\
\hline S Kainji & 0 & 2 & 0 & 0 & 0 & 0 & 0 & 0 & 0 & 2 \\
\hline S Kalamu & 0 & 1 & 0 & 0 & 0 & 0 & 2 & 0 & 0 & 3 \\
\hline$S$ Kedougou & 2 & 0 & 0 & 0 & 0 & 0 & 0 & 0 & 0 & 2 \\
\hline S Kentucky & 0 & 7 & 0 & 0 & 0 & 0 & 0 & 0 & 0 & 7 \\
\hline S Kiambu & 1 & 4 & 0 & 0 & 0 & 0 & 0 & 0 & 0 & 5 \\
\hline$S$ Kingston & 0 & 1 & 0 & 0 & 0 & 0 & 0 & 0 & 0 & 1 \\
\hline S Kotu & 0 & 3 & 0 & 0 & 0 & 0 & 0 & 0 & 0 & 3 \\
\hline
\end{tabular}


TABLE 1 (Continues...): Number of isolates and zoological distribution of the various serotypes 2007-2014.

\begin{tabular}{|c|c|c|c|c|c|c|c|c|c|c|}
\hline Serotype & Cattle & Poultry & Leopard & Rhino & Crocodile & Feline & Equine & Sheep and goat & Pig & Total \\
\hline S Kottbus & 1 & 0 & 0 & 0 & 0 & 0 & 0 & 0 & 0 & 1 \\
\hline S Kouka & 0 & 2 & 0 & 0 & 0 & 0 & 0 & 0 & 0 & 2 \\
\hline$S$ Lexington & 0 & 1 & 0 & 0 & 0 & 0 & 0 & 0 & 0 & 1 \\
\hline$S$ Lockleaze & 0 & 2 & 0 & 0 & 0 & 0 & 0 & 0 & 0 & 2 \\
\hline$S$ London & 0 & 4 & 0 & 0 & 0 & 0 & 0 & 0 & 0 & 4 \\
\hline S Mandera & 0 & 0 & 0 & 0 & 0 & 0 & 1 & 0 & 0 & 1 \\
\hline S Manhattan & 0 & 1 & 0 & 0 & 0 & 0 & 2 & 0 & 0 & 3 \\
\hline S Mbandaka & 0 & 6 & 0 & 0 & 0 & 0 & 0 & 0 & 0 & 6 \\
\hline S Minnesota & 0 & 25 & 0 & 0 & 0 & 0 & 0 & 0 & 0 & 25 \\
\hline S Mikawasima & 0 & 0 & 6 & 0 & 0 & 0 & 0 & 0 & 0 & 6 \\
\hline S Montevideo & 0 & 10 & 0 & 0 & 0 & 0 & 0 & 0 & 0 & 10 \\
\hline S Muenchen & 2 & 43 & 0 & 0 & 0 & 0 & 2 & 1 & 0 & 48 \\
\hline S Newport & 0 & 22 & 0 & 0 & 0 & 0 & 0 & 0 & 0 & 22 \\
\hline SOhio & 0 & 3 & 0 & 0 & 0 & 0 & 0 & 0 & 0 & 3 \\
\hline SOslo & 0 & 1 & 0 & 0 & 0 & 0 & 0 & 0 & 0 & 1 \\
\hline SOthmarschen & 1 & 2 & 0 & 0 & 0 & 0 & 0 & 0 & 0 & 3 \\
\hline S Paratyphi & 0 & 5 & 0 & 0 & 0 & 0 & 0 & 0 & 0 & 5 \\
\hline S Planckendael & 0 & 1 & 0 & 0 & 0 & 0 & 0 & 0 & 0 & 1 \\
\hline$S$ Poitiers & 0 & 1 & 0 & 0 & 0 & 0 & 0 & 0 & 0 & 1 \\
\hline$S$ Panama & 0 & 1 & 0 & 0 & 0 & 0 & 0 & 0 & 0 & 1 \\
\hline S Poona & 0 & 1 & 0 & 0 & 0 & 0 & 0 & 0 & 0 & 1 \\
\hline$S$ Pretoria & 2 & 3 & 0 & 0 & 0 & 0 & 2 & 0 & 0 & 7 \\
\hline S Reading & 2 & 2 & 0 & 0 & 0 & 0 & 0 & 0 & 0 & 4 \\
\hline$S$ Rideau & 0 & 0 & 0 & 0 & 0 & 0 & 1 & 0 & 0 & 1 \\
\hline$S$ Rissen & 0 & 5 & 0 & 0 & 0 & 0 & 0 & 0 & 0 & 5 \\
\hline$S$ Ruiru & 0 & 4 & 0 & 0 & 0 & 0 & 0 & 0 & 0 & 4 \\
\hline S Saintpaul & 0 & 11 & 0 & 0 & 0 & 0 & 0 & 0 & 0 & 11 \\
\hline S Salford & 1 & 0 & 0 & 0 & 0 & 0 & 0 & 0 & 0 & 1 \\
\hline S Schoeneberg & 1 & 0 & 0 & 0 & 0 & 0 & 2 & 0 & 0 & 3 \\
\hline S Schwarzengrund & 0 & 47 & 0 & 0 & 0 & 0 & 3 & 0 & 0 & 50 \\
\hline S Senftenberg & 0 & 25 & 0 & 0 & 0 & 0 & 0 & 0 & 0 & 25 \\
\hline S Shubra & 0 & 2 & 0 & 0 & 0 & 0 & 0 & 0 & 0 & 2 \\
\hline S Stanley & 0 & 1 & 0 & 0 & 0 & 0 & 0 & 0 & 0 & 1 \\
\hline S Stanleyville & 0 & 13 & 0 & 0 & 0 & 0 & 0 & 0 & 0 & 13 \\
\hline S Stratford & 1 & 0 & 0 & 0 & 0 & 0 & 0 & 0 & 0 & 1 \\
\hline S Tallahassee & 0 & 2 & 0 & 0 & 0 & 0 & 0 & 0 & 0 & 2 \\
\hline$S$ Tennessee & 0 & 7 & 0 & 0 & 0 & 0 & 0 & 0 & 0 & 7 \\
\hline S Thompson & 0 & 13 & 0 & 0 & 0 & 0 & 0 & 0 & 0 & 13 \\
\hline$S$ Tsevie & 0 & 2 & 0 & 0 & 0 & 0 & 0 & 0 & 0 & 2 \\
\hline S Typhimurium & 17 & 66 & 0 & 6 & 0 & 4 & 35 & 9 & 9 & 146 \\
\hline S Virchow & 0 & 13 & 0 & 0 & 0 & 0 & 0 & 0 & 0 & 13 \\
\hline SWinneba & 0 & 6 & 0 & 0 & 0 & 0 & 0 & 0 & 0 & 6 \\
\hline S Wippra & 0 & 4 & 0 & 0 & 0 & 0 & 0 & 0 & 0 & 4 \\
\hline$S$ Yoruba & 0 & 1 & 0 & 0 & 0 & 0 & 0 & 2 & 0 & 3 \\
\hline Total & 116 & 871 & 6 & 22 & 8 & 6 & 162 & 26 & 16 & 1233 \\
\hline
\end{tabular}

Sixteen isolations involving five serotypes were recorded in pigs. The majority of isolates were $S$. Typhimurium, that accounted for $56.3 \%$ of the cases, followed by $S$. Enteritidis $(18.7 \%)$ and $S$. Derby (12.5\%) (Table 6). In captive and wild animals, 42 isolates involving 6 serotypes were recorded (Table 7). Salmonella Heidelberg (33.33\%) and S. Typhimurium $(23.8 \%)$ were the two major serotypes isolated during the survey period. Salmonella Fulda (19.0\%) and S. Mikawasima (14.3\%) were isolated exclusively from crocodiles and leopards, respectively (Table 7). Salmonella Dublin (2.4\%) and S. Enteritidis (7.1\%) were detected from rhinoceroses.

\section{Discussion}

Laboratory diagnosis continues to provide important epidemiological information that contributes significantly to continuous disease surveillance programmes in the country, if properly validated tests are used. The data set presented in this report is a follow-up of similar laboratory surveillance information carried out from 1996 through 2006 as described elsewhere (Kidanemariam et al. 2010).

There has been a steady increase over the years in the number of Salmonella serotypes isolated and in the number of animal 
TABLE 2: Common Salmonella serotypes in poultry in South Africa.

\begin{tabular}{|c|c|c|c|c|c|c|c|c|c|}
\hline Isolate & 2007 & 2008 & 2009 & 2010 & 2011 & 2012 & 2013 & 2014 & Total \\
\hline \multicolumn{10}{|c|}{$S$ Enteritidis } \\
\hline Count & 26 & 14 & 10 & 62 & 2 & 12 & 31 & 2 & 159 \\
\hline$\%$ & 16.88 & 14.00 & 10.30 & 42.42 & 6.89 & 7.69 & 19.25 & 8.33 & 18.25 \\
\hline \multicolumn{10}{|c|}{$S$ Heidelberg } \\
\hline Count & 20 & 0 & 11 & 5 & 0 & 63 & 34 & 0 & 133 \\
\hline$\%$ & 12.98 & 0.00 & 11.34 & 3.42 & 0.00 & 40.38 & 21.11 & 0.00 & 15.26 \\
\hline Count & 1 & 6 & 5 & 4 & 8 & 3 & 8 & 6 & 41 \\
\hline$\%$ & 0.64 & 6.00 & 5.15 & 2.73 & 27.58 & 1.92 & 4.96 & 25.00 & 4.70 \\
\hline \multicolumn{10}{|c|}{$S$ Minnesota } \\
\hline Count & 2 & 6 & 1 & 3 & 0 & 4 & 9 & 0 & 25 \\
\hline$\%$ & 1.29 & 6.00 & 1.03 & 2.05 & 0.00 & 2.56 & 5.59 & 0.00 & 2.87 \\
\hline \multicolumn{10}{|c|}{$S$ Muenchen } \\
\hline Count & 2 & 15 & 0 & 2 & 0 & 17 & 7 & 0 & 43 \\
\hline \multicolumn{10}{|c|}{ S Newport } \\
\hline Count & 0 & 5 & 3 & 0 & 3 & 5 & 3 & 3 & 22 \\
\hline$\%$ & 0.00 & 5.00 & 3.09 & 0.00 & 10.34 & 3.20 & 1.86 & 12.50 & 2.52 \\
\hline \multicolumn{10}{|c|}{ S Schwarzengrund } \\
\hline Count & 0 & 5 & 2 & 8 & 2 & 5 & 18 & 7 & 47 \\
\hline$\%$ & 0.00 & 5.00 & 2.06 & 5.47 & 6.89 & 3.20 & 11.18 & 29.16 & 5.39 \\
\hline \multicolumn{10}{|c|}{$S$ Senftenberg } \\
\hline Count & 3 & 1 & 15 & & 5 & 0 & 1 & 0 & 25 \\
\hline$\%$ & 1.94 & 1.00 & 15.46 & 0.00 & 17.24 & 0.00 & 0.62 & 0.00 & 2.87 \\
\hline \multicolumn{10}{|c|}{$S$ Typhimurium } \\
\hline Count & 22 & 8 & 20 & 18 & 1 & 9 & 7 & 0 & 66 \\
\hline$\%$ & 14.28 & 8.00 & 20.61 & 12.32 & 3.44 & 5.76 & 4.34 & 0.00 & 7.57 \\
\hline \multicolumn{10}{|l|}{ Others } \\
\hline Count & 78 & 48 & 34 & 44 & 16 & 41 & 43 & 6 & 310 \\
\hline Count & 154 & 100 & 97 & 146 & 29 & 156 & 161 & 24 & 871 \\
\hline$\%$ & 100.00 & 100.00 & 100.00 & 100.00 & 100.00 & 100.00 & 100.00 & 100.00 & 100.00 \\
\hline
\end{tabular}

TABLE 3: Common Salmonella serotypes in cattle in South Africa.

\begin{tabular}{|c|c|c|c|c|c|c|c|c|c|}
\hline Isolate & 2007 & 2008 & 2009 & 2010 & 2011 & 2012 & 2013 & 2014 & Total \\
\hline \multicolumn{10}{|l|}{$S$ Anatum } \\
\hline Count & 1 & 5 & 1 & 0 & 0 & 5 & 1 & 0 & 13 \\
\hline$\%$ & 5.00 & 27.77 & 6.66 & 0.00 & 0.00 & 33.33 & 4.00 & 0.00 & 11.20 \\
\hline \multicolumn{10}{|l|}{$S$ Dublin } \\
\hline Count & 8 & 2 & 6 & 9 & 3 & 2 & 6 & 3 & 39 \\
\hline$\%$ & 40.00 & 11.11 & 40.00 & 60.00 & 75.00 & 13.33 & 24.00 & 75.00 & 33.60 \\
\hline$S$ Infantis & & & & & & & & & \\
\hline Count & 1 & 2 & 0 & 0 & 0 & 2 & 3 & 0 & 8 \\
\hline$\%$ & 5.00 & 11.11 & 0.00 & 0.00 & 0.00 & 13.33 & 12.00 & 0.00 & 6.89 \\
\hline \multicolumn{10}{|c|}{$S$ Typhimurium } \\
\hline Count & 7 & 4 & 1 & 1 & 0 & 3 & 1 & 0 & 17 \\
\hline$\%$ & 35.00 & 22.22 & 6.66 & 6.66 & 0.00 & 20.00 & 4.00 & 0.00 & 14.65 \\
\hline \multicolumn{10}{|l|}{ Others } \\
\hline Count & 3 & 5 & 7 & 5 & 1 & 3 & 14 & 1 & 39 \\
\hline \multicolumn{10}{|l|}{ Total } \\
\hline Count & 20 & 18 & 15 & 15 & 4 & 15 & 25 & 4 & 116 \\
\hline$\%$ & 100.00 & 100.00 & 100.00 & 100.00 & 100.00 & 100.00 & 100.00 & 100.00 & 100.00 \\
\hline
\end{tabular}

species involved. Salmonella serotypes clearly seem to differ in their distributions between different animal populations (Table 1). For instance, approximately $95.3 \%$ of all detected Salmonella serotypes are predominantly associated with domestic species, namely poultry, cattle, horses and sheep and goats, whereas rhinoceroses, leopards, crocodiles and pigs contributed only $4.7 \%$. However, care must be taken when considering these data because of the possibility of a skewed focus from targeted sampling of mostly food animals as compared to wild and game animals. In spite of this, the data probably give a reasonable assessment of the incidence of salmonellosis and the Salmonella serotypes involved. 
TABLE 4: Common Salmonella serotypes in horses in South Africa. $\dagger$

\begin{tabular}{|c|c|c|c|c|c|c|c|}
\hline Isolate & 2008 & 2009 & 2011 & 2012 & 2013 & 2014 & Total \\
\hline \multicolumn{8}{|c|}{ S Achersleben } \\
\hline Count & 0 & 0 & 1 & 0 & 0 & 1 & 2 \\
\hline$\%$ & 0.00 & 0.00 & 1.72 & 0.00 & 0.00 & 2.12 & 1.23 \\
\hline \multicolumn{8}{|c|}{ S Adeoyo } \\
\hline Count & 0 & 0 & 0 & 0 & 9 & 0 & 9 \\
\hline$\%$ & 0.00 & 0.00 & 0.00 & 0.00 & 20.45 & 0.00 & 5.56 \\
\hline \multicolumn{8}{|c|}{$S$ Anatum } \\
\hline Count & 4 & 0 & 8 & 4 & 12 & 8 & 36 \\
\hline$\%$ & 100.00 & 0.00 & 13.79 & 100.00 & 27.27 & 17.02 & 22.22 \\
\hline \multicolumn{8}{|c|}{$S$ Colorado } \\
\hline Count & 0 & 0 & 2 & 0 & 0 & 2 & 4 \\
\hline$\%$ & 0.00 & 0.00 & 3.45 & 0.00 & 0.00 & 4.25 & 2.47 \\
\hline \multicolumn{8}{|c|}{$S$ Glasgow } \\
\hline Count & 0 & 0 & 0 & 0 & 2 & 0 & 2 \\
\hline \multicolumn{8}{|c|}{$S$ Gustaria } \\
\hline Count & 0 & 0 & 0 & 0 & 1 & 0 & 1 \\
\hline$\%$ & 0.00 & 0.00 & 0.00 & 0.00 & 2.27 & 0.00 & 0.62 \\
\hline \multicolumn{8}{|c|}{$S$ Heidelberg } \\
\hline Count & 0 & 0 & 32 & 0 & 0 & 20 & 52 \\
\hline$\%$ & 0.00 & 0.00 & 55.17 & 0.00 & 0.00 & 42.55 & 32.1 \\
\hline \multicolumn{8}{|l|}{$S$ Infantis } \\
\hline Count & 0 & 0 & 0 & 0 & 5 & 0 & 5 \\
\hline$\%$ & 0.00 & 0.00 & 0.00 & 0.00 & 11.36 & 0.00 & 3.08 \\
\hline \multicolumn{8}{|l|}{$S$ Kalamu } \\
\hline Count & 0 & 0 & 1 & 0 & 0 & 2 & 2 \\
\hline$\%$ & 0.00 & 0.00 & 1.72 & 0.00 & 0.00 & 4.25 & 1.23 \\
\hline \multicolumn{8}{|c|}{$S$ Mandera } \\
\hline Count & 0 & 0 & 0 & 0 & 1 & 0 & 1 \\
\hline Count & 0 & 0 & 1 & 0 & 0 & 1 & 2 \\
\hline$\%$ & 0.00 & 0.00 & 1.72 & 0.00 & 0.00 & 2.12 & 1.23 \\
\hline \multicolumn{8}{|c|}{$S$ Muenchen } \\
\hline Count & 0 & 0 & 1 & 0 & 0 & 1 & 2 \\
\hline$\%$ & 0.00 & 0.00 & 1.72 & 0.00 & 0.00 & 2.12 & 1.23 \\
\hline \multicolumn{8}{|c|}{$S$ Pretoria } \\
\hline Count & 0 & 0 & 0 & 0 & 2 & 0 & 2 \\
\hline$\%$ & 0.00 & 0.00 & 0.00 & 0.00 & 4.54 & 0.00 & 1.23 \\
\hline \multicolumn{8}{|l|}{$S$ Rideau } \\
\hline Count & 0 & 0 & 0 & 0 & 1 & 0 & 1 \\
\hline$\%$ & 0.00 & 0.00 & 0.00 & 0.00 & 2.27 & 0.00 & 0.61 \\
\hline \multicolumn{8}{|l|}{ S Sambre } \\
\hline Count & 0 & 0 & 0 & 0 & 1 & 0 & 1 \\
\hline$\%$ & 0.00 & 0.00 & 0.00 & 0.00 & 2.27 & 0.00 & 0.61 \\
\hline \multicolumn{8}{|c|}{ S Schoeneberg } \\
\hline Count & 0 & 0 & 1 & 0 & 0 & 1 & 2 \\
\hline$\%$ & 0.00 & 0.00 & 1.72 & 0.00 & 0.00 & 2.12 & 1.23 \\
\hline S Schwar & & & & & & & \\
\hline Count & 0 & 1 & 0 & 0 & 2 & 0 & 3 \\
\hline$\%$ & 0.00 & 16.70 & 0.00 & 0.00 & 4.54 & 0.00 & 1.85 \\
\hline$S$ Typhim & & & & & & & \\
\hline Count & 0 & 5 & 11 & 0 & 8 & 11 & 35 \\
\hline$\%$ & 0.00 & 83.33 & 18.96 & 0.00 & 18.18 & 23.40 & 21.60 \\
\hline Total & & & & & & & \\
\hline Count & 4 & 6 & 58 & 4 & 44 & 47 & 162 \\
\hline$\%$ & 100.00 & 100.00 & 100.00 & 100.00 & 100.00 & 100.00 & 100.00 \\
\hline
\end{tabular}

$\dagger$, No samples were presented for testing between 2007 and 2010.

Over 2500 Salmonella serovars are recognised worldwide (Coburn et al. 2007; EFSA 2008), and the number continues to rise. However, despite the existence of formidable number of different serotypes, only a few are commonly associated with clinical disease in humans and animals (Warnick et al. 2001). It is also noted in the current study that despite the 
TABLE 5: Common Salmonella serotypes in sheep and goats in South Africa.

\begin{tabular}{|c|c|c|c|c|}
\hline Isolate & 2010 & 2012 & 2013 & Total \\
\hline \multicolumn{5}{|l|}{ S Agona } \\
\hline Count & 0 & 1 & 1 & 2 \\
\hline$\%$ & 0.00 & 14.28 & 7.69 & 7.69 \\
\hline \multicolumn{5}{|l|}{$S$ Dublin } \\
\hline Count & 0 & 0 & 2 & 2 \\
\hline$\%$ & 0.00 & 0.00 & 15.38 & 7.69 \\
\hline \multicolumn{5}{|c|}{$S$ Enteritidis } \\
\hline Count & 1 & 1 & 2 & 4 \\
\hline$\%$ & 16.66 & 14.28 & 15.38 & 15.38 \\
\hline \multicolumn{5}{|l|}{$S$ Infantis } \\
\hline Count & 0 & 0 & 3 & 3 \\
\hline$\%$ & 0.00 & 0.00 & 23.07 & 11.53 \\
\hline \multicolumn{5}{|l|}{$S$ Isangi } \\
\hline Count & 1 & 2 & 0 & 3 \\
\hline \multicolumn{5}{|c|}{$S$ Muenchen } \\
\hline Count & 0 & 1 & 0 & 1 \\
\hline$\%$ & 0.00 & 14.28 & 0.00 & 3.84 \\
\hline \multicolumn{5}{|c|}{$S$ Typhimurium } \\
\hline Count & 3 & 1 & 5 & 9 \\
\hline$\%$ & 50.00 & 14.28 & 38.46 & 34.61 \\
\hline \multicolumn{5}{|l|}{$S$ Yoruba } \\
\hline Count & 1 & 1 & 0 & 2 \\
\hline$\%$ & 16.66 & 14.28 & 0.00 & 7.69 \\
\hline \multicolumn{5}{|l|}{ Total } \\
\hline Count & 6 & 7 & 13 & 26 \\
\hline$\%$ & 100.00 & 100.00 & 100.00 & 100.00 \\
\hline
\end{tabular}

TABLE 6: Common Salmonella serotypes in pigs in South Africa.

\begin{tabular}{|c|c|c|c|c|c|c|c|c|}
\hline Isolate & 2008 & 2009 & 2010 & 2011 & 2012 & 2013 & 2014 & Total \\
\hline \multicolumn{9}{|l|}{ S Derby } \\
\hline Count & 1 & 0 & 0 & 0 & 1 & 0 & 0 & 2 \\
\hline$\%$ & 20.00 & 0.00 & 0.00 & 0.00 & 20.00 & 0.00 & 0.00 & 12.50 \\
\hline \multicolumn{9}{|c|}{$S$ Enteritidis } \\
\hline Count & 1 & 0 & 0 & 0 & 1 & 1 & 0 & 3 \\
\hline$\%$ & 20.00 & 0.00 & 0.00 & 0.00 & 20.00 & 25.00 & 0.00 & 18.75 \\
\hline \multicolumn{9}{|l|}{$S$ Give } \\
\hline Count & 0 & 0 & 0 & 0 & 0 & 1 & 0 & 1 \\
\hline$\%$ & 0.00 & 0.00 & 0.00 & 0.00 & 0.00 & 25.00 & 0.00 & 6.25 \\
\hline \multicolumn{9}{|c|}{$S$ Sandiego } \\
\hline Count & 0 & 0 & 1 & 0 & 0 & 0 & 0 & 1 \\
\hline$\%$ & 0.00 & 0.00 & 100.00 & 0.00 & 0.00 & 0.00 & 0.00 & 6.25 \\
\hline \multicolumn{9}{|c|}{$S$ Typhimurium } \\
\hline Count & 3 & 0 & 0 & 0 & 3 & 2 & 1 & 9 \\
\hline$\%$ & 60.00 & 0.00 & 0.00 & 0.00 & 60.00 & 50.00 & 100.00 & 56.25 \\
\hline \multicolumn{9}{|l|}{ Total } \\
\hline Count & 5 & 0 & 1 & 0 & 5 & 4 & 1 & 16 \\
\hline$\%$ & 100.00 & 0.00 & 100.00 & 0.00 & 100.00 & 100.00 & 100.00 & 100.00 \\
\hline
\end{tabular}

large number of serotypes involved, the majority of isolates were mainly represented by a small number of serotypes. For instance, from the total of 108 serotypes detected among 1229 isolates, only 8 serotypes contributed $63.0 \%$ (775 isolates), while 100 serotypes collectively accounted for $37.0 \%$ (454 isolates).

Salmonella serotypes can be divided into host-specific, hostadapted and generalist serotypes, with important implications for the epidemiology and risk factors of the diseases in the associated host species (Baumler et al. 1998; Uzzau et al. 2000).
Host-specific serotypes, such as S. Paratyphi A in humans and S. Gallinarum biovars Gallinarum and Pullorum in chickens, only caused disease in one host species (Pascopella et al. 1995; Uzzau et al. 2000). These host-specific serotypes were not isolated during the survey period. Salmonella Paratyphi A is exclusively a human pathogen, and samples are submitted to public health facilities. Nonetheless, S. Typhi was confirmed through blood and stool cultures from cases of a typhoid fever outbreak in South Africa (Anon 2016). The absence of $S$. Gallinarum and S. Pullorum in the data set of the current survey could be because of the regular targeted 
TABLE 7: Common Salmonella serotypes in wild and captive animals in South Africa. $\dagger$

\begin{tabular}{|c|c|c|c|c|c|}
\hline Isolate & 2011 & 2012 & 2013 & 2014 & Total \\
\hline \multicolumn{6}{|l|}{$S$ Dublin } \\
\hline Count & 0 & 0 & 0 & 1 & 1 \\
\hline \multicolumn{6}{|c|}{$S$ Enteritidis } \\
\hline Count & 1 & 0 & 1 & 1 & 3 \\
\hline$\%$ & 5.00 & 0.00 & 100.00 & 4.76 & 7.14 \\
\hline \multicolumn{6}{|l|}{$S$ Fulda } \\
\hline Count & 4 & 0 & 0 & 4 & 8 \\
\hline$\%$ & 20.00 & 0.00 & 0.00 & 19.04 & 19.04 \\
\hline \multicolumn{6}{|c|}{ S Mikawasima } \\
\hline Count & 3 & 0 & 0 & 3 & 6 \\
\hline$\%$ & 15.00 & 0.00 & 0.00 & 14.28 & 14.28 \\
\hline \multicolumn{6}{|c|}{$S$ Heidelberg } \\
\hline$\%$ & 35.00 & 0.00 & 0.00 & 33.33 & 33.33 \\
\hline \multicolumn{6}{|c|}{$S$ Typhimurium } \\
\hline Count & 5 & 0 & 0 & 5 & 10 \\
\hline$\%$ & 25.00 & 0.00 & 0.00 & 23.81 & 23.81 \\
\hline \multicolumn{6}{|l|}{ Total } \\
\hline Count & 20 & 0 & 1 & 21 & 42 \\
\hline$\%$ & 100.00 & 0.00 & 100.00 & 100.00 & 100.00 \\
\hline
\end{tabular}

$\dagger$, No samples were presented for testing between 2007 and 2010.

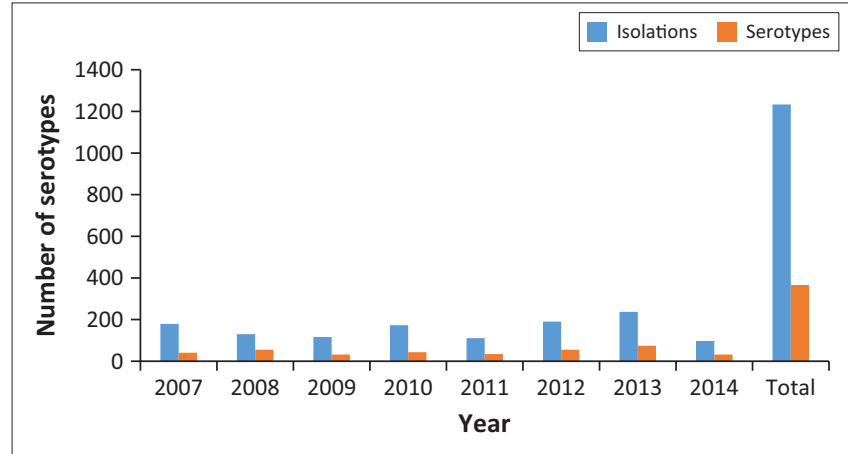

FIGURE 1: Annual Salmonella isolations and number of serotypes from animal origin.

monitoring programme that assisted poultry farms to control and prevent Salmonella infections in general and infections as a result of $S$. Gallinarum and $S$. Pullorum in particular (A.K. Gelaw, unpublished results). In addition, small incidences are less likely to be reported and to be passed unnoticed. Furthermore, the lack of either serovar in the current survey may also be attributed to the selection criteria in the methodology that often targets motile salmonellae.

Host-adapted serotypes are predominantly associated with a single host species, but reports suggest that these serotypes can cause disease in other species as well (Pascopella et al. 1995; Uzzau et al. 2000). For example, S. Dublin, S. Enteritidis and $S$. Choleraesuis were previously regarded as hostadapted serotypes for cattle, chicken and pigs, respectively (Anderson et al. 1997; Uzzau et al. 2000). However, it became apparent that these serotypes commonly cause disease in a broad range of hosts and may be considered as generalist serotypes. This is supported by the results of the current survey where $S$. Dublin was isolated from cattle, goats and rhinoceroses, and S. Enteritidis was isolated from poultry, pigs, sheep and rhinoceroses, demonstrating the ability of these serotypes to adapt and infect multiple hosts.

Generalist serotypes such as Salmonella Typhimurium, on the other hand, are associated with a wide range of hosts. The current data supported this assertion, as S. Typhimurium was detected across a wide range of animal species that include poultry, cattle, horses, sheep, goats and pigs (Table 1). This serotype was also found in wild and captive animals such as a leopard, rhinoceros and crocodile. Similar studies have demonstrated that $S$. Typhimurium is the most common serovar isolated in livestock (Morgan et al. 2004; Rabsch et al. 2002) and human non-typhoidal infections, especially in immune-compromised patients (Calvert, Stewart \& Reilly 1998; Gordon 2008; Keddy et al. 2017; Voetsch et al. 2004).

Salmonella Heidelberg is one of the generalist serotypes and was by far the most common serotype, representing $16.2 \%$ of the total isolations, with the majority originating from horses, in which species it accounted for $32.1 \%$ of the total cases. This result could partially be attributed to the increased epidemiological monitoring to ascertain the causes of clinical episodes of enteric infections in the hospitalised horses at the Veterinary Academic Hospital of the University of Pretoria (J. Gouws, pers. com., June 2015). Faecal material was submitted for testing, and $S$. Heidelberg was isolated from horses admitted to the hospital. Because of the potential risk of spread of Salmonella from an infected horse, the equine hospital has introduced biosecurity measures to help prevent disease transmission to personnel, the environment and other patients. Similar reports showed that hospitalised horses were more likely to shed Salmonella than horses housed in their own barns, possibly because of stress or 
illness (Amavisit et al. 2001). A study at a large veterinary hospital in the United States demonstrated that $13 \%$ of horses admitted for colic were shedding Salmonella species (Kim et al. 2001).

Salmonella Typhimurium exhibited the widest zoological distribution (Table 1). This finding is not unexpected because $S$. Typhimurium has been thought of as the most ubiquitous and broadest host-range serotype, as it is frequently associated with diseases in numerous host species, including humans, livestock, domestic fowl, rodents and birds (Bahnson et al. 2006; Padungtod, Kadohira \& Hill 2008). It was, however, demonstrated in the current survey that the incidence of $S$. Typhimurium was relatively higher in poultry and equines with incidence rates of $45.2 \%$ and $23.9 \%$, respectively.

Although S. Enteritidis was isolated from various host species, it was by far the most common serotype encountered in poultry, accounting for $93.5 \%$ of the incidents. The remaining $6.5 \%$ were shared among cattle $(0.6 \%)$, rhinoceros $(1.7 \%)$, sheep $(2.3 \%)$ and pigs $(1.7 \%)$. This serotype is among the most common pathogens of chicken that could also adversely affect the health of human beings and other animal species following exposures (Kidanemariam et al. 2010). Vertical transmission with internal transovarian contamination of egg-yolk with $S$. Enteritidis has been confirmed, making uncooked eggs no longer safe to eat (Altekruse et al. 1993). Furthermore, S. Enteritidis was confirmed as causes of human outbreaks of non-typhoidal salmonellosis associated with the consumption of foods of animal origin in South Africa (Muvhali et al. 2017). Salmonella Enteritidis is often presented separately from other serotypes of Salmonella because this bacterium is specifically cited in zoonosis control legislation. The South African government has included S. Enteritidis in the list of controlled diseases (Anon 1984; Kidanemariam et al. 2010).

Salmonella Dublin and Salmonella Typhimurium are the two predominant serotypes detected in cattle in the current survey, accounting for $48.3 \%$ of the total recorded isolations. The relative incidence of $S$. Dublin $(33.6 \%)$ is, however, higher than that of $S$. Typhimurium (14.6\%) for the survey period. Similar studies have shown that $S$. Dublin may potentially be the most frequently isolated serotype in cattle, more than the broad host-range serotypes, such as S. Typhimurium (Brackelsberg, Nolan \& Brown 1997; Kidanemariam et al. 2010). It should be noted that the disease epidemiology of $S$. Dublin and $S$. Typhimurium varies considerably. Most importantly, cattle infected with $S$. Dublin often become asymptomatic carriers and continue to excrete large numbers of organisms in their faeces for many years and often for life (Giles, Hopper \& Wray 1989; Rice, Besser \& Hancock 1997; Vanselow et al. 2007). Effective control of infection should thus include removal of chronically infected cattle and implementation of stringent biosecurity measures. On the other hand, hosts infected with $S$. Typhimurium will only shed the organism for a few weeks to a month after clinical recovery (Huston, Wittum \& Love 2002). However, it should be noted that $S$. Typhimurium tends to persist in the environment for longer periods (Rabsch et al. 2002). One study estimated the median duration of shedding in dairy cattle to equal 50 days, with a maximum duration of 391 days (Cummings et al. 2009). This can be mitigated by implementing proper biosecurity measures at all times.

Clinically affected herds and certain management systems may pose an increased risk to public health. Little is known about the Salmonella risk posed to humans by indirect animal contact, especially through environmental contamination. Considering the public health significance of this group of bacteria, further studies, especially on spatial clustering of human cases around livestock premises, are needed to assess the indirect risks posed by livestock operations in South Africa.

\section{Acknowledgements}

This work was supported partly by a grant from the Agricultural Research Council - Onderstepoort Veterinary Research (P10000046). We acknowledge Mrs Maluleka for the technical support during data retrieval. We are also indebted to Dr Sabeta for his critical comments on the manuscript.

\section{Competing interests}

The authors declare that there is no conflict of interest with regard to the writing of this article.

\section{Authors' contributions}

A.K.G. contributed to the study design, interpretation of data and preparation of the manuscript. I.M. and P.N. contributed to laboratory testing and data collection.

\section{References}

Altekruse, S.F., Bauer, N., Chanlongbutra, A., DeSagun, R., Naugle, A., Schlosser, W. et al., 2006, 'Salmonella Enteritidis in broiler chickens, United States, 2000-2005', Emerging Infectious Diseases 12(12), 1848-1852. https://doi.org/10.3201/ eid1212.060653

Altekruse, S., Koehler, J., Hickman-Brenner, F., Tauxe, R.V. \& Ferris, K., 1993, 'A comparison of Salmonella Enteritidis phage types from egg-associated outbreaks
and implicated laying flocks', Epidemiology and Infection 110, 17-22. https://doi. and implicated laying flocks', Epide
org/10.1017/S0950268800050639

Amavisit, P., Markham, P.F., Lightfoot, D., Whithear, K.G. \& Browning, G.F., 2001, 'Molecular epidemiology of Salmonella Heidelberg in an equine hospital', Veterinary Microbiology 80, 85-98.

Anderson, R.L., Walker, R.L., Hird, D.W. \& Blanchard, P.C., 1997, 'Case-control study of an outbreak of clinical disease attributable to Salmonella infection in eight dairy herds', Journal of the American Veterinary Medical Association 210, 528-530. https://doi.org/10.1016/S0378-1135(00)00373-4

Bahnson, P.B., Fedorka-Cray, P.J., Ladely, S.R. \& Mateus-Pinilla, N.E., 2006, 'Herd-level risk factors for Salmonella enterica subsp. enterica in U.S. market pigs', Preventive Veterinary Medicine 76(3-4), 249-262. https://doi.org/10.1016/j.prevetmed. 2006.05.009

Baumler, A.J., Tsolis, R.M., Ficht, T.A. \& Adams, L.G., 1998, 'Evolution of host adaptation in Salmonella enterica', Infection and Immunity 66(10), 4579-4587.

Brackelsberg, C.A., Nolan, L.K. \& Brown, J., 1997, 'Characterization of Salmonella Dublin and Salmonella Typhimurium (Copenhagen) isolates from cattle', Veterinary Research Communications 21(6), 409-420. https://doi.org/10.1023/ A:1005803301827

Calvert, N., Stewart, W.C. \& Reilly, W.J., 1998, 'Salmonella Typhimurium DT104 infection in people and animals in Scotland: A collaborative epidemiological study 1993-96', Veterinary Record 143(13), 351-354. https://doi.org/10.1136/ vr.143.13.351 
Coburn, B., Grassl, G.A. \& Finlay, B.B., 2007, 'Salmonella, the host and disease: A brief review', Immunology and Cell Biology 85, 112-118. https://doi.org/10.1038/sj. icb.7100007

Cummings, K.J., Warnick, L.D., Alexander, K.A., Cripps, C.J., Grohn, Y.T., James, K.L. et al., 2009, 'The duration of fecal Salmonella shedding following clinical disease among dairy cattle in the northeastern USA', Preventive Veterinary Medicine 92 134-139. https://doi.org/10.1016/j.prevetmed.2009.07.002

Department of Agriculture, Forestry \& Fisheries, 1984, Animal Diseases Act 1984 (Act 35 of 1984), Republic of South Africa.

EFSA (European Food Safety Authority), 2008, 'Scientific opinion of the panel on biological hazards on a request from the European Commission on a quantitative microbiological risk assessment on Salmonella in meat: Source attribution for human salmonellosis from meat', EFSA Journal 625, 1-32.

Giles, N., Hopper, S.A. \& Wray, C., 1989, 'Persistence of S. Typhimurium in a large dairy herd', Epidemiology and Infection 103(2), 235-241. https://doi.org/10.1017/ S0950268800030582

Gordon, M., 2008, 'Salmonella infections in immunocompromised adults', Journal of Infections 56, 413-422. https://doi.org/10.1016/j.jinf.2008.03.012

Grimont, P.A. \& Weill, F.-X., 2007, Antigenic formulae of the Salmonella Serovars, 9th edn., WHO Collaborating Centre for Reference and Research on Salmonella, edn., WHO Collaboratin
Institut Pasteur, Paris.

Huston, C.L., Wittum, T.E. \& Love, B.C., 2002, 'Persistent fecal Salmonella shedding in five dairy herds', Journal of the American Veterinary Medical Association 220(5), 650-655. https://doi.org/10.2460/javma.2002.220.650

Keddy, K.H., Takuva, S., Musekiwa, A., Puren, A.J., Sooka, A., Karstaedt, A. et al. 2017, 'An association between decreasing incidence of invasive non-typhoida salmonellosis and increased use of antiretroviral therapy, Gauteng Province, South Africa, 2003-2013', PLoS One 12(3), e0173091. https://doi:10.1371/ journal.pone.0173091

Kidanemariam, A., Engelbrecht, M. \& Picard, J., 2010, 'Retrospective study on the incidence of Salmonella isolations in animals in South Africa, 1996 to 2006 Journal of the South African Veterinary Association 81(1), 37-44. https://doi. org/10.4102/jsava.v81i1.94

Kim, L.M., Morley, P.S., Traub-Dargatz, J.L., Salman, M.D. \& Gentry-Weeks, C., 2001 'Factors associated with Salmonella shedding among equine colic patients at a veterinary teaching hospital', Journal of the American Veterinary Medical Association 218, 740-748. https://doi.org/10.2460/javma.2001.218.740

Kimura, A.C., Reddy, V., Marcus, R., Cieslak, P.R., Mohle-Boetani, J.C. \& Kassenborg, H.D., 2004, 'Chicken consumption is a newly identified risk factor for sporadic Salmonella enterica serotype Enteritidis infections in the United States: A casecontrol study in FoodNet sites', Clinical Infectious Diseases 38, 244-252. https:// doi.org/10.1086/381576

Mead, P.S., Slutsker, L., Dietz, V., McCaig, L.F., Bresee, J.S., Shapiro, C. et al., 1999 'Food-related illness and death in the United States', Emerging Infectious Diseases 5(5), 607-625. https://doi.org/10.3201/eid0505.990502

Morgan, E., Campbell, J.D., Rowe, S.C., Bispham, J., Stevens, M.P., Bowen, A.J. et al, 2004 , 'Identification of host-specific colonization factors of Salmonella enterica serovar Typhimurium', Molecular Microbiology 54, 994-1010. https://doi. org/10.1111/j.1365-2958.2004.04323.x
Muvhali, M., Smith, A.M., Rakgantso, A.M. \& Keddy, K.H., 2017, 'Investigation of Salmonella Enteritidis outbreaks in South Africa using multi-locus variable number tandem repeats analysis, 2013-2015', BMC Infectious Diseases 17(1), 661. https:// tandem repeats analysis, 2013-2015',
doi.org/10.1186/s12879-017-2751-8

National Institute of Communicable Diseases, Communicable Diseases Communique, 2016, 'Salmonella Typhi cases in South Africa, 2016', vol. 15(2), p. 1, Sandringham, Republic of South Africa.

Padungtod, P., Kadohira, M. \& Hill, G., 2008, 'Livestock production and foodborne diseases from food animals in Thailand', Journal of Veterinary Medical Science 70(9), 873-879. https://doi.org/10.1292/jvms.70.873

Pascopella, L., Raupach, B., Ghori, N., Monack, D., Falkow, S. \& Small, P.L., 1995, 'Host restriction phenotypes of Salmonella Typhi and Salmonella Gallinarum', Infection and Immunity 63, 4329-4335.

Peek, S.F., Hartmann, F.A., Thomas, C.B. \& Nordlund, K.V., 2004, 'Isolation of Salmonella spp from the environment of dairies without any history of clinical salmonellosis', Journal of the American Veterinary Medical Association 225(4), 574-577. https://doi.org/10.2460/javma.2004.225.574

Popoff, M.Y., 2001, Antigenic formula of the Salmonella serovars, 8th edn., WHO Collaborating Centre for Reference and Research on Salmonella, Institut Pasteur, Paris.

Rabsch, W., Andrewes, H.L., Kingsley, R.A., Prager, R., Tschäpe, H., Adams, L.G. et al., 2002 , 'Salmonella enterica serotype Typhimurium and its host adapted variants', Infection and Immunity 70, 2249-2255. https://doi.org/10.1128/IAl.70.5.2249Infection and 2255.2002

Refsum, T., Heir, E., Kapperud, G., Vardund, T. \& Holstad, G., 2002, 'Molecular epidemiology of Salmonella enterica serovar Typhimurium isolates determined by pulsed-field gel electrophoresis: Comparison of isolates from avian wildlife, domestic pulsed-field gel electrophoresis: Comparison of isolates from avian wild life, domestic animals, and the environment in Norway', Applied and Environmental Microt
$68(11), 5600-5606$. https://doi.org/10.1128/AEM.68.11.5600-5606.2002

Rice, D.H., Besser, T.E. \& Hancock, D.D., 1997, 'Epidemiology and virulence assessment of Salmonella Dublin', Veterinary Microbiology 56, 111-124. https://doi. of Salmonella Dublin', Veterinary
$\mathrm{org} / 10.1016 / \mathrm{S} 0378-1135(96) 01352-1$

Uzzau, S., Brown, D.J., Wallis, T., Rubino, S., Leori, G., Bernard, S. et al., 2000, 'Host adapted serotypes of Salmonella enterica', Epidemiology and Infection 125(2), 229-255. https://doi.org/10.1017/S0950268899004379

Vanselow, B.A., Hum, S., Hornitzky, M.A., Eamens, G.J. \& Quinn, K., 2007, 'Salmonella Typhimurium persistence in a Hunter Valley dairy herd', Australian Veterinary Journal 85(11), 446-450. https://doi.org/10.1111/j.1751-0813.2007.00224.x

Veling, J., Wilpshaar, H., Frankena, K., Bartels, C. \& Barkema, H., 2002, 'Risk factors for clinical Salmonella enterica subsp. enterica serovar Typhimurium infection on Dutch dairy farms', Preventive Veterinary Medicine 54(2), 157-168. https://doi. org/10.1016/S0167-5877(02)00023-5

Voetsch, A.C., Van Gilder, T.J., Angulo, F.J., Farley, M.M., Shallow, S., Marcus, R. et al. 2004, 'FoodNet estimate of the burden of illness caused by non-typhoidal Salmonella infections in the United States', Clinical Infectious Disease 38, 127-134. https://doi.org/10.1086/381578

Warnick, L.D., Crofton, L.M., Pelzer, K.D. \& Hawkins, M.J., 2001, 'Risk factors for clinical salmonellosis in Virginia, USA, cattle herds', Preventive Veterinary Medicine 49 259-275. https://doi.org/10.1016/S0167-5877(01)00172-6 